\title{
Patient Characteristics, Procedural and Safety Outcomes of Bariatric Surgery in England: a Retrospective Cohort Study-2006-2012
}

\author{
Sun Sun ${ }^{1,2,3}$ (D) Oleg Borisenko ${ }^{1}$ Tim Spelman ${ }^{1,4,5} \cdot$ Ahmed R. Ahmed $^{6}$
}

Published online: 26 October 2017

(C) The Author(s) 2017. This article is an open access publication

\begin{abstract}
Background The objective of the study is to analyze procedural and safety outcomes associated with bariatric surgery and describe the characteristics of patients undertaking bariatric procedures in England between April 2006 and March 2012.

Methods This is a retrospective cohort study of all adult patients in England diagnosed with obesity and undergoing bariatric surgery as a primary procedure in NHS-funded sites between April 2006 and March 2012 using data sourced from the Hospital Episode Statistics dataset. Length of stay (LOS), 30-day readmission, and postsurgery complication were analyzed as primary outcomes. Socio-demographic background, provider type, procedure volume, and comorbidities were all analyzed as potential explanatory variables.

Results Gastric bypass (GBP, 12,628) was the most utilized procedure, followed by gastric banding $(\mathrm{GB}, 6872)$ and sleeve gastrectomy (SG, 3251). The most prevalent comorbidity was type 2 diabetes (23\%). Inpatient mortality was low $(\leq 0.15 \%)$
\end{abstract}

Sun Sun

sun.sun@synergus.com

1 Synergus AB, Kevinge Strand 20, 18257 Stockholm, Sweden

2 Health Outcomes and Economic Evaluation Research Group, Center for Healthcare Ethics, Department of Learning, Information, Management and Ethics, Karolinska Institutet, Stockholm, Sweden

3 Division of Epidemiology and Global Health, Department of Public Health and Clinical Medicine, Umeå University, Umeå, Sweden

4 Centre for Population Health, Burnet Institute, Melbourne, Australia

5 School of Public Health and Preventive Medicine, Monash University, Melbourne, Australia

6 Imperial College London, London, UK for all procedure types. LOS and the risks of both postoperative complication and 30-day readmission were significantly lower for GB, relative to those for GBP and SG. Ethnicity, geographical area, surgery type, and volume were all associated with LOS, risk of readmission, and complication. Provider type and deprivation were further associated with LOS while age correlated with readmission only. An increasing comorbidity burden was associated with an increased risk of both readmission and complication.

Conclusions Gastric bypass was the most frequently reported procedure in England across the observation period. While utilization across all procedure types increased between 2007 and 2010, overall uptake of bariatric surgery in England represents only a small proportion of the eligible population. Readmission and complication rates were lower for gastric banding relative to those for either gastric bypass or sleeve gastrectomy. The observed inpatient mortality rate was low across all procedure types.

Keywords Morbid obesity · Bariatric surgery $\cdot$ Hospital data . England $\cdot$ Length of stay $\cdot$ Health outcomes

\section{Background}

Obesity and overweight are global health problems associated with significant morbidity and mortality [1]. The World Health Organization (WHO) estimates that approximately $25 \%$ of women and $22 \%$ of men in Europe were obese in 2014 [2]. This represents a 2 and 3\% increase from 2010 figures for females and males, respectively [2]. The obesity rate in England has more than doubled within the past 20 years from $12 \%$ in 1993 to $25 \%$ in 2014 [3]. The current rate of $25 \%$ is the second highest in Europe after Hungary (29\% in 2014) [4]. The prevalence of morbid obesity in particular (defined as 
a body mass index (BMI) of at least $40 \mathrm{~kg} / \mathrm{m}^{2}$, or BMI $\geq 35 \mathrm{~kg} /$ $\mathrm{m}^{2}$ plus at least one obesity-related complication [5]) has also increased markedly from $1.4 \%$ of females and $0.2 \%$ of males in 1993 to 3.8 and $1.8 \%$ of female and males, respectively in 2014 [3]. Obesity increases the risk of both developing and worsening a large range of associated diseases and comorbidities, including type 2 diabetes mellitus (T2DM) [6, 7], cardiovascular disorders [8,9], and cancers (particularly endometrial, post-menopausal breast, and colorectal) [10].

Management of obesity involves first-line lifestyle modification including diet, physical activity, and behavioral therapy $[5,11]$. This may be supplemented with adjunct pharmacotherapy, which currently includes agents such as orlistat and liraglutide in the UK $[5,11]$. Sustained weight loss is typically defined as a total $5-10 \%$ loss of body weight, sustained for a minimum of 6 months [12]. These conventional treatments are only partially efficacious in achieving sustained weight loss [13]. In contrast, bariatric surgery has been observed to be more effective in reducing weight in both the short- and long-term among morbidly obese patients relative to nonsurgical interventions [12-16]. Importantly, in the context of a patient's overall comorbidity load, bariatric surgery has also been reported to improve obesity-related comorbidity [17]. Recent analyses of the Hospital Episode Statistics (HES) dataset [18] and the National Bariatric Surgery Registry (NBSR) [19] both reported increased utilization of bariatric surgery. HES reported an increase from 72 to 347 procedures in England between 1996 and 2004 [18] while NBSR reported an increase from approximately 500 to 6500 procedures between 2006 and 2013 across the broader UK and Ireland [19]. This increase was most marked within middle-aged women. A 2010 analysis of HES data reported a marked increase in the number of bariatric procedures performed in England from 238 in 2000 to 2543 in 2007 [19]. However, this observation of an increasing trend ceased in 2011 and was replaced with a steady reduction in HES-reported bariatric procedures in HES between 2011 and 2013 (8794, 8024, and 6384 procedures, respectively) [20].

Analysis of NBSR data observed both a low inpatient mortality rate $(<0.1 \%)$ and complication rate $(<3 \%)$ following the primary procedure. This compares well with best practice international mortality benchmarks (30-day mortality: Sweden $0.05 \%$ [21], Italy $0.2 \%$ [22], US $0.3 \%$ [23]). Bariatric management of obesity is thus generally considered to be safe. Furthermore, the average post-operative stay has been observed to be no more than 3 days; NBSR reported that for 2011-2013 majority of patients stayed 3 days or less in the hospital after operation (GBP and SG 77\%; GB 86\%), supporting efficient use of inpatient resources [19].

The objective of this study was to analyze procedural and safety outcomes associated with bariatric surgery and describe the characteristics of patients undertaking bariatric procedures in England between April 2006 and March 2012.

\section{Methods}

\section{Patients}

Data were obtained from the HES Admitted Care database. HES collects patient-level data from all admissions, accident and emergency attendances, and outpatient appointments at National Health Service (NHS) hospitals across England, in addition to centers funded by the NHS, which can include a subset of independent providers [24]. Each HES record captures clinical, demographic, administrative, and geographical information linked to the individual episode of care. Diagnosis codes used in HES are based on the International Classification of Disease version 10 (ICD10) [25]. For each admission episode, patients are assigned a primary diagnosis code and up to 19 associated secondary diagnoses. Patients aged 18 years or above who were diagnosed with obesity (ICD-10: E66.0 - obesity due to excess calories, E66.1 — drug-induced obesity, E66.2-extreme obesity and hypoventilation, E66.8 - other obesity, E66.9 - obesity unspecified) and underwent bariatric surgery as a primary procedure in NHS sites or NHS-funded sites between April 2006 and March 2012 were included in the analysis. HES is a patient administrative dataset, and a strict statistical disclosure control is applied in accordance with the HES protocol and all data is anonymized [24].

\section{Surgery Type}

Patients were coded to one of four primary procedure groups based on OPCS-4 codes: GBP, SG, GB, and other procedures (including gastroplasty and duodenal switch). The insertion of gastric balloons was not considered. The full list of OPCS-4 and ICD-10 codes for all procedures and diagnoses is summarized in Table 1.

\section{Outcomes}

The primary outcomes of this study were length of stay (LOS), 30-day readmission, and surgery complication. LOS was defined as the duration of the episode of care (or "spell") in days. Readmission was defined as an emergency readmission of a post-operative patient within 30 days of discharge following admission for a bariatric surgical procedure. Complications related to surgery were defined by clinical experts, based on secondary diagnosis codes (ICD-10) as further detailed in Table 1. It mainly includes complications of procedures (T81); mechanical complication of gastrointestinal prosthetic devices, implants, and grafts (T855); other complications of surgical and medical care, not elsewhere classified (T88); misadventures to patients during surgical and medical care (Y60-Y69); anesthesiology devices associated with adverse incidents (Y70); gastroenterology and urology devices 
Table 1 OPCS-4 and ICD-10 codes used in the analysis

\begin{tabular}{|c|c|}
\hline Type of bariatric surgery & OPCS-4 codes \\
\hline Gastric bypass & $\begin{array}{l}\text { G281, G282, G283, G288, G289, G310, G311, G312, G314, G316, G318, G319, } \\
\text { G320, G321, G323, G325, G328, G329, G330, G331, G333, G338, G339, G271, } \\
\text { G274, G275, G279, G313, G322, G324, G332, G335, G336, G717 }\end{array}$ \\
\hline Sleeve gastrectomy & $\mathrm{G} 284, \mathrm{G} 285$ \\
\hline Gastric banding & G303 \\
\hline Other & $\begin{array}{l}\text { G018, G022, G023, G024, G025, G028, G029, G032, G033, G034, G035, G036, } \\
\text { G038, G272, G273, G278, G301, G302, G304, G308, G309, G315, G481, G482, } \\
\text { G485, G486, G491, G492, G493, G498, G499, G513, G716 } \\
\text { ICD-10 codes }\end{array}$ \\
\hline Procedure complications & T81, T855, T88, Y60-Y70, Y73, Y74, Y81-Y84 \\
\hline \multicolumn{2}{|l|}{ Obesity-related comorbidity } \\
\hline $\begin{array}{l}\text { Abnormal glucose } \\
\text { tolerance }\end{array}$ & $\mathrm{R} 73.0$ \\
\hline $\begin{array}{l}\text { Degenerative joint } \\
\text { disease }\end{array}$ & M15-M19 \\
\hline Depression & $\mathrm{F} 32, \mathrm{~F} 33$ \\
\hline Diabetes type 2 & E11 \\
\hline Dyslipidemia & E78 \\
\hline Gallstone & K80 \\
\hline $\begin{array}{l}\text { Gastroesophageal } \\
\text { reflux disease }\end{array}$ & K21 \\
\hline Hypertension & I11, I15 \\
\hline Infertility & N97 \\
\hline Obstructive sleep apnea & G47.3 \\
\hline
\end{tabular}

associated with adverse incidents (Y73); general hospital and personal-use devices associated with adverse incidents (Y74); general- and plastic-surgery devices associated with adverse incidents (Y81); other and unspecified medical devices associated with adverse incidents (Y82); surgical and other medical procedures as the cause of abnormal reaction of the patient, or of later complication, without mention of misadventure at the time of the procedure (Y83-84). Inpatient mortality was analyzed as a secondary outcome and was defined as a hospital discharge status of death. Weight and body mass index (BMI) were not available from the HES dataset and thus were unable to be analyzed, as either an outcome or explanatory variable. Similarly, data around patient selection and referral for surgery were likewise unavailable from HES.

\section{Variables and Definitions}

Age was divided into five groups: 18-34 years, 35-44 years, 45-54 years, 55-64 years, and 65 years and above. Ethnic group was defined as either "Caucasian" or "nonCaucasian." Social and economic disadvantage was quantified using the index of multiple deprivation (IMD) as a continuous variable [26]. Patients were then categorized into five groups based on the quintiles of IMD. Geographical area was based on a regional HES code as follows: London, North England (North West, North East, Yorkshire, and the
Humber), Central England (West and East midlands), East England, South England (South West and South East), and other. Procedure volume was calculated according to the total number of procedures carried out at each provider during the study period. Procedure volume was categorized into mutually exclusive quartiles and defined as either "very low" (1-399 procedures), "low" (400-848), "medium" (849-1231), or "high" (1231-1537) volume, consistent with the approach adopted by previous studies [27-29]. Provider type was defined as either NHS trust provider or independent provider. Obesity-related comorbidity was defined based on ICD-10 codes detailed as summarized in Table 1. Cumulative comorbidity burden was captured using the Charlson comorbidity index (CCI) [30].

\section{Statistical Analyses}

Categorical variables were summarized using frequency and percentage. Continuous variables were first assessed for skewness using a Shapiro-Wilk test and summarized using mean and standard deviation (SD) or median and inter-quartile range (IQR) as appropriate. Ordinary least squares (OLS) linear regression of the mean was used to model log-transformed LOS $\left(\log _{10}(L O S+1)\right)$, secondary to its significant skew. Logistic regression was used for a model of the binary readmission and complication outcome variables. For each 
outcome, adjusted regression models were derived using stepwise forward selection of candidate explanatory variables. Overall goodness-of-fit was examined using the adjusted $R^{2}$ for the linear mean regression and an adjusted McFadden pseudo $R^{2}$ for the logistic modelling. The final adjusted model included both the primary explanatory variable and other significant explanatory variables. Utilization data was analyzed for the observation period for which full-year data was available (i.e., 2007-2011). For all analyses, $p<0.05$ was considered significant. All analyses were undertaken in $\mathrm{R}$ version $3.2 .2[31]$.

\section{Results}

\section{Patient Characteristics}

A total of 26,420 patients were included in the analysis. Gastric bypass (GBP) was the most frequently reported procedure $(48 \%, 12,658)$, followed by GB $(26 \%, 6872)$ and SG $(12 \%, 3251)$ (Table 2). Mean age at surgery was the highest among patients undergoing SG (45.6 (10.4)). Females accounted mostly across all procedure types and were the highest for GB (80\%) and lowest for SG (69\%). The highest proportion of procedures were performed in North England (27\%, 7231 procedures), followed by South England (24\%, 6249 procedures), London (23\%, 6078 procedures), Central
England (18\%, 4788 procedures), and East England (6\%, 1649 procedures).

Fifty-four percent of patients were associated with a low IMD. Median (IQR) procedure volume (defined as the count of procedures per provider) was the highest for GBP at 898 $(565,1274)$ and lowest for GB at $821(388,1184)$.

Most procedures reported in HES were performed by NHS trust providers, accounting for $99 \%$ of GB, $97 \%$ of SG, and $92 \%$ of GBP, with the remainder reported by a small subset of NHS-funded independent providers.

The most frequently reported obesity-related comorbidity across all procedures was T2DM, followed by obstructive sleep apnea, dyslipidemia, depression, degenerative joint disease, and gastroesophageal reflux disease. The percentage of patients having no comorbidities was broadly comparable across all procedure types (64\% for GB, $62 \%$ for GBP, $61 \%$ for $\mathrm{SG})$.

\section{Utilization Trends}

Utilization trends across 2007 through 2011 by sex, age group, and geographical area are summarized in Figs. 1, 2, and 3, respectively. Females account for a greater proportion of total procedures (18,656 procedures total across a 5-year period compared with 5730 in males) (Fig. 1). The number of bariatric procedures has increased for both sexes across the 2007 to 2010 period; however, a reduction from 2010 to 2011 was observed for females only.

Table 2 Conditions for Charlson comorbidity index (CCI)

\begin{tabular}{|c|c|c|}
\hline Conditions & ICD-10 codes & Weight* \\
\hline Acute myocardial infarction & $\mathrm{I} 21, \mathrm{I} 22, \mathrm{I} 23, \mathrm{I} 252, \mathrm{I} 258$ & 5 \\
\hline Cerebral vascular accident & G450, G451, G452, G454, G458, G459, G46, I60-I69 & 11 \\
\hline Congestive heart failure & I50 & 13 \\
\hline Connective tissue disorder & M05, M060, M063, M069, M32, M332, M34, M353 & 4 \\
\hline Dementia & F00, F01, F02, F03, F051 & 14 \\
\hline Diabetes & $\begin{array}{l}\text { E101, E105, E106, E108, E109, E111, E115, E116, E118, } \\
\text { E119, E131, E136, E138, E139, E141, E145, E146, E148, E149 }\end{array}$ & 3 \\
\hline Liver disease & K702, K703, K717, K73, K74 & 8 \\
\hline Peptic ulcer & K25, K26, K27, K28 & 9 \\
\hline Peripheral vascular disease & I71, I739, I790, R02, Z958, Z959 & 6 \\
\hline Pulmonary disease & J40-J47, J60-J67 & 4 \\
\hline Cancer & $\mathrm{C} 00-\mathrm{C} 76, \mathrm{C} 81-\mathrm{C} 97$ & 8 \\
\hline Diabetes complications & $\begin{array}{l}\text { E102, E103, E104, E107, E112, E113, E114, E117, E132, E133, } \\
\text { E134, E137, E142, E143, E144, E147 }\end{array}$ & 1 \\
\hline Paraplegia & G041, G81, G820, G821, G822 & 1 \\
\hline Renal disease & I12, I13, N01, N03, N052-N056, N072-N074, N18, N19, N25 & 10 \\
\hline Metastatic cancer & C77, C78, C79, C80 143 & 14 \\
\hline Severe liver disease & K721, K729, K766, K767 183 & 18 \\
\hline HIV & B20, B21, B22, B23, B24, O987 26 & 2 \\
\hline
\end{tabular}

*If any secondary diagnosis fields contain any condition for both cancer and metastatic cancer, additional 8 weights will be deducted from CCI; if $\mathrm{CCI}<0$, then set $\mathrm{CCI}$ is re-coded as 0 
Fig. 1 Bariatric surgery utilization trends over time, by sex (year 2006 and 2012 data were excluded from the figure, as the data were not available for the whole year)
6000

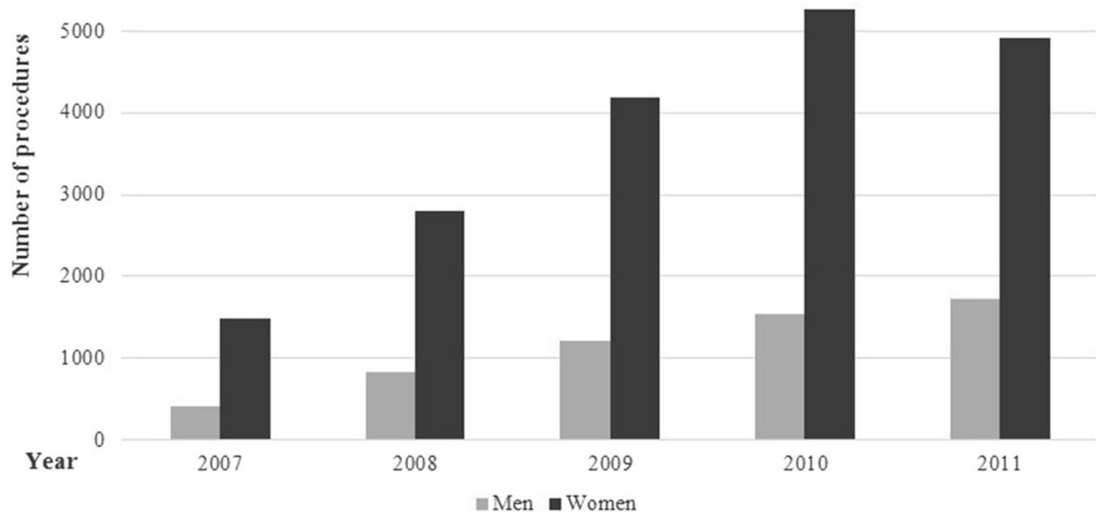

The number of procedures increased steadily over the 2007 to 2010 intervals across all age groups. The rate remained stable between 2010 and 2011 for most age groups except that for patients aged between 45 and 64 years where a reduction was observed (Fig. 2). Patients aged between 35 and 44 years accounted for the highest proportion of procedures (7972 procedures total across a 5 -year period). The total number of procedures was the smallest in the 65 years and above age group. Furthermore, the total number of procedures increased across all geographical areas between 2007 and 2010 . However, between 2010 and 2011, this increase was only sustained in the North and East England areas, with London and South and Central England all reporting a reduction (Fig. 3). The greatest proportion of procedures were performed in North England (6748 procedures across the 5-year period), followed by South England (5808), London (5498), and Central England (4412), while East England performed the least number of procedures (1522).

\section{Outcome Analysis}

The primary outcomes differed significantly across by procedure type (Table 3 ). Readmission rates (6\% for GB, $10 \%$ for
GBP and SG) and complication rates (1\% for GB, $4 \%$ for GBP and SG) were lower for GB relative to either those for GBP or SG. The median (IQR) for LOS was shorter for GB (1 $(1,2))$, relative to either that for $\operatorname{GBP}(3(2,4))$ or SG $(3(2,4))$. Inpatient mortality was low for all types of procedures $(0.15 \%$ for GBP, $0.12 \%$ for SG, and $0 \%$ for GB); however, there were no significant differences across different procedure types. The results for the adjusted (multivariate) regression analyses of the primary LOS, 30-day readmission, and post-surgery complication end points are summarized in Table 4.

\section{Length of Stay}

Medium procedure volume was associated with a $0.04 \mathrm{log}$ unit ( 0.1 day) decrease in LOS ( $\beta$-coefficient -0.040 ; CI $-0.0486,-0.0322$ ) relative to very low volume (Table 5). High volume was associated with a $0.07 \log$ unit $(0.18$ day $)$ increase in $\operatorname{LOS}$ ( $\beta$ 0.072; CI 0.063, 0.081). Independent providers were associated with a $0.06 \log$ unit $(0.15$ days) decrease in $\operatorname{LOS}(\beta-0.062$; CI $-0.076,-0.047)$, relative to the NHS trust providers. Geographical location, IMD, and ethnicity were also associated with LOS. However, while these marginal differences were statistically significant, they
Fig. 2 Bariatric surgery utilization trends over time, by age group (year 2006 and 2012 data were excluded from the figure, as the data were not available for the whole year)

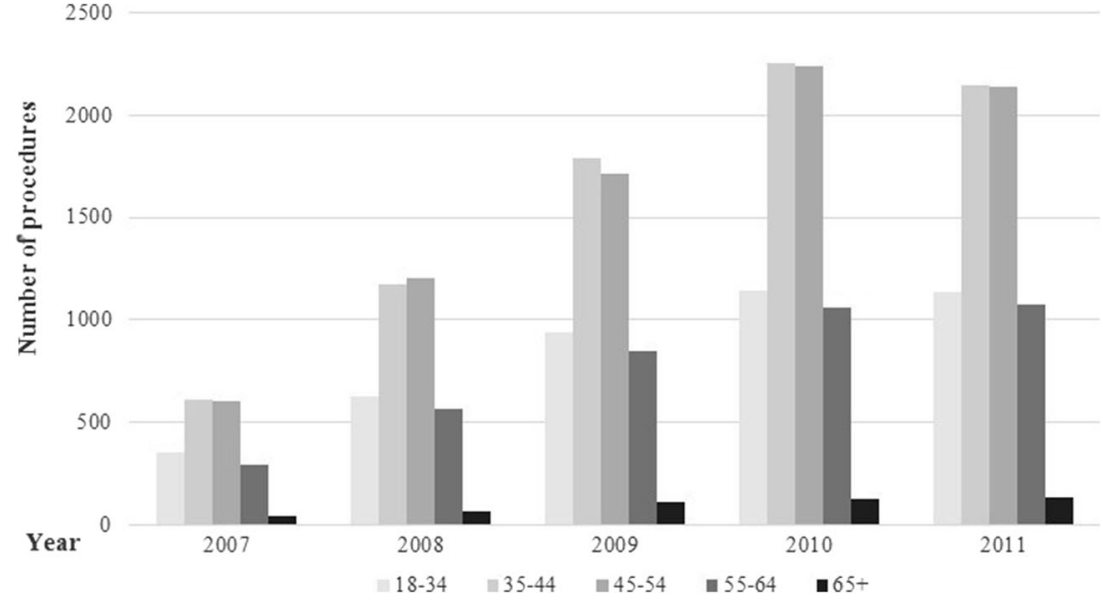


Fig. 3 Bariatric surgery utilization trends over time, by geographical area (year 2006 and 2012 data were excluded from the figure, as the data were not available for the whole year)

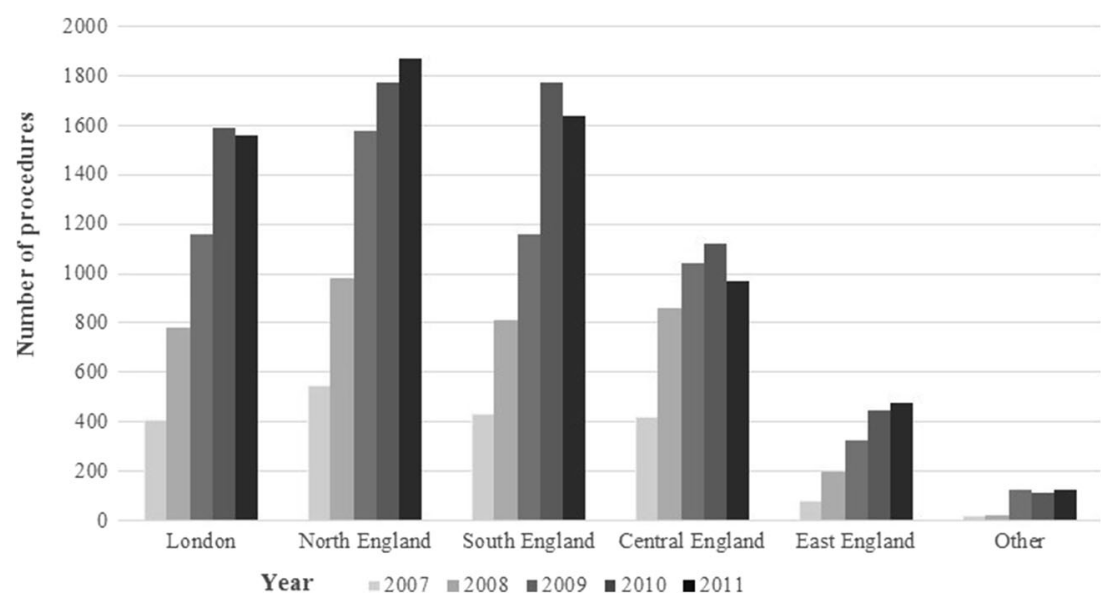

were too small to represent clinical significance. Both GB $(\beta-0.087$; CI $-0.094,-0.080)$ and SG $(\beta-0.009 ; \mathrm{CI}$ $-0.019,-0.0003)$ were associated with small decreases in LOS relative to GBP, adjusting for all other model covariates; however, these associations were similarly not clinically significant. There was no association between LOS and either age, sex, or CCI.

\section{Thirty-Day Readmission}

Only a high procedure volume (OR 0.784; CI 0.684, 0.899) was associated with significantly lower odds of readmission relative to very low volume. A high CCI (OR 1.649; CI 1.433, 1.891) was associated with higher odds of readmission, relative to "no comorbidity". Non-Caucasians were associated with significantly lower odds of readmission relative to Caucasians (OR 0.821; CI 0.745, 0.904). Relative to London, East (OR 0.767; CI 0.616, 0.950) and South England (OR 0.769; CI 0.672, 0.890) had significantly lower odds of readmission. The odds of readmission in East and Central England did not differ significantly from that in London. All age groups except for age group 45-54 years were inversely correlated with odds of readmission with the oldest patients (aged 65 years or above) associated with the lowest odds (OR 0.685; CI 0.482, 0.949). GB was associated with significantly lower odds of 30-day readmission relative to GBP (OR 0.579; CI 0.514, 0.652), adjusting for all other model covariates. There was no significant difference in risk of readmission between SG and GBP. There was no association between readmission and sex, deprivation, or provider type.

\section{Post-surgery Complications}

Medium procedure volume was associated with significantly lower odds of post-operative complication relative to very low volume (OR 0.618; CI 0.488, 0.780), while neither low nor high volume differed from very low volume. Both moderate
(OR 1.477; CI 1.265, 1.724) and high (OR 1.866; CI 1.479, 2.333) CCI were associated with significantly higher odds of post-surgery complication, relative to no comorbidity. NonCaucasians were associated with significantly lower odds of post-surgery complication relative to Caucasians (OR 0.714; CI $0.599,0.848)$. GB was associated with significantly lower odds of post-surgery complication relative to GBP (OR 0.275 ; CI $0.212,0.351$ ), adjusting for all other model covariates. There was no significant difference in the risk of postsurgery complication between SG and GBP. There was no association between risk of post-surgery complication and age, sex, geographical area, provider type, or deprivation.

\section{Discussion}

Based on NICE guidelines, approximately two million people in England would presently qualify for bariatric surgery [32]. However, the actual number of bariatric procedures undertaken nationally each year represents only a small proportion of the eligible population, with the population-standardized bariatric surgical rate in England of 117 per million of the population being comparatively low relative to that in other European settings [33]. Furthermore, the relative distribution of procedure types in England was far more mixed. While GBP was the most frequently reported procedure across the observation period (48\%), this was considerably smaller than the proportions reported in Sweden, Denmark, and Belgium where GBP clearly dominates $(98,96$, and $80 \%$, respectively) [33]. By contrast, GB was used relatively frequently in England (26\%), with only France (19\%) and Italy (37\%) reporting comparable utilization levels [33]. While overall utilization across all procedure types increased across 2007-2010, the late decline in bariatric procedures between 2010 and 2011 was primarily driven by a reduction in the number of GB procedures undertaken. A recent analysis of the NBSR supports this observation, albeit 
Table 3 Patient characteristics, by type of surgery

Variables

Surgery type

Gastric bypass

$(n=12,628)$

Sleeve gastrectomy

Gastric banding

Other

Individual characteristics

Age, mean (SD)

$44.3(10.1)$

$45.6(10.4)$

$44.3(10.7)$

$44.0(10.4)$

Age group, $n(\%)$

18-34 years

2138 (17)

$513(16)$

1247 (18)

676 (19)

35-44 years

934 (29)

$2276(33)$

1203 (33)

45-54 years

1149 (35)

2083 (30)

$1140(31)$

55-64 years

$570(18)$

1068 (16)

$555(15)$

65 years and above

85 (3)

Female sex, $n(\%)$

$2242(69)$

$198(3)$

65 (2)

Geographical area, $n(\%)$

North England

$9743(77)$

$5478(80)$

$2690(74)$

Central England

$4173(33)$

754 (23)

1002 (15)

1302 (36)

East England

1200 (9)

390 (12)

2188 (32)

1010 (28)

South England

255 (8)

3251 (26)

495 (15)

81 (1)

65 (2)

1237 (38)

2200 (32)

303(8)

London

2640 (21)

1285 (19)

$916(25)$

Other

146 (1)

116 (2)

43 (1)

Caucasian ethnicity, $n(\%)$

120 (4)

Index of multiple deprivation (IMD), $n(\%)$

1 st quintile (most deprived)

8759 (69)

2149 (66)

4613 (67)

2726 (75)

3874 (31)

1016 (32)

1751 (26)

1317 (37)

3077 (25)

815 (26)

1567 (23)

925 (26)

2414 (19)

552 (18)

1345 (20)

610 (17)

1830 (15)

423 (14)

1124 (17)

462 (13)

$1311(10)$

322 (10)

966 (14)

281 (8)

Clinical characteristics

Provider type, $n(\%)$

NHS trust provider

Independent provider

Procedure volume, median (IQR)

\begin{tabular}{llll}
$11,659(92)$ & $3166(97)$ & $6826(99)$ & $3517(97)$ \\
$999(8)$ & $85(3)$ & $46(1)$ & $122(3)$ \\
$898(565,1274)$ & $848(605,1131)$ & $821(388,1184)$ & $709(388,1184)$ \\
$3006(24)$ & & & \\
$2803(22)$ & $633(19)$ & $1929(28)$ & $1210(33)$ \\
$3618(29)$ & $1295(40)$ & $1574(23)$ & $945(26)$ \\
$3227(26)$ & $650(20)$ & $2589(38)$ & $703(19)$ \\
$3004(24)$ & $670(21)$ & $778(11)$ & $778(21)$ \\
$2016(16)$ & & & $854(23)$ \\
$1206(10)$ & $780(24)$ & $1595(23)$ & $584(16)$ \\
$1061(8)$ & $511(16)$ & $1038(15)$ & $349(10)$ \\
$822(6)$ & $306(9)$ & $672(10)$ & $295(8)$ \\
$603(5)$ & $295(9)$ & $581(8)$ & $219(6)$ \\
$7832(62)$ & $186(6)$ & $447(7)$ & $178(5)$ \\
$3846(30)$ & $169(5)$ & $329(5)$ & $2265(62)$ \\
$980(8)$ & & & $1101(30)$ \\
& $1983(61)$ & $4428(64)$ & $273(8)$ \\
\hline $949(29)$ & $1965(29)$ &
\end{tabular}

Very low

Low

Medium

High

Comorbidity, $n$ (\%)

T2DM

Obstructive sleep apnea

Dyslipidemia

Depression

Degenerative joint disease

Gastroesophageal reflux disease

Charlson comorbidity index (CCI), $n(\%)$

No comorbidity (0)

Moderate (1-5)

High ( $\geq 6)$
122 (3)

$1210(33)$

$945(26)$

03 (19)

$854(23)$

584 (16)

$295(8)$

219 (6)

$2265(62)$

$273(8)$ 
Table 4 Surgery outcomes, by type of surgery complication outcomes

\begin{tabular}{|c|c|c|c|c|}
\hline & \multicolumn{4}{|l|}{ Surgery type } \\
\hline & $\begin{array}{l}\text { Gastric bypass } \\
(n=12,628)\end{array}$ & $\begin{array}{l}\text { Sleeve gastrectomy } \\
\quad(n=3251)\end{array}$ & $\begin{array}{c}\text { Gastric banding } \\
(n=6872)\end{array}$ & $\begin{array}{l}\text { Other } \\
\qquad(n=3639)\end{array}$ \\
\hline Inpatient mortality, $n(\%)$ & $19(0.15)$ & $4(0.12)$ & $0(0)$ & $2(0.05)$ \\
\hline Readmission, $n(\%)$ & $1279(10)$ & $334(10)$ & $443(6)$ & $498(14)$ \\
\hline Complications, $n(\%)$ & $494(4)$ & $123(4)$ & $76(1)$ & $114(3)$ \\
\hline Length of stay (days), median (IQR) & $3(2,4)$ & $3(2,4)$ & $1(1,2)$ & $2(2,4)$ \\
\hline
\end{tabular}

over a longer time period, reporting an overall decline from $40 \%$ in 2007 to $15 \%$ in 2013 [19].

Our study observed that LOS and the risk of both postoperative complication and readmission were significantly lower for GB. While this likely reflects the less invasive nature of banding, studies of long-term outcomes up to 15 years postsurgery in GB patients have reported relatively low success rates in terms of sustained weight loss [34]. Of the patient factors assessed, comorbidity level, deprivation index, and ethnicity were all associated with the study outcomes. There was no association between gender and any of the outcomes tested. However, older age at the time of procedure was associated with a reduction in the risk of any readmission. Neither the NICE guidelines nor precedent literature support older age ( $>65$ years) in itself as a contraindication for surgery [35-37]. However, this diverse range of both patient and provider factors observed in our analysis to associate with post-operative outcome, independent of the procedure type, supports existing guidelines recommending case-by-case assessment of older bariatric surgery candidates [35].

With regard to provider factors, NHS-funded procedures undertaken by independent providers were associated with a significant, albeit marginal, reduction in LOS relative to NHS trust providers on adjusted modelling. LOS varied with geography while the odds of readmission were significantly lower in East and South England relative to London, independent of provider volume. No significant differences in the risk of postoperative complication were found across the different geographical areas. An increasing provider-level procedure volume was also associated with a decreasing risk of readmission. Post-operative complication was also significantly lower for medium procedure volume relative to very low volume providers. This observed link between provider factors and postoperative course supports arguments for expanding minimum standards around infrastructure and volume, in order to optimize post-surgical outcomes of surgery [38, 39]. The International Federation for the Surgery of Obesity and Metabolic Disorders (IFSO) recommends at least 100 surgical cases per year including revisional cases per qualified provider [38]. Similarly, the American Society for Bariatric Surgery (ASBS) requires at least 125 bariatric surgical cases per year per qualified provider, with each surgeon performing a minimum of 50 cases per year and having a total experience of at least 125 cases as the primary surgeon [40, 41]. However, no recommendations regarding minimum number of surgeon and unit volumes are presently available from relevant professional surgical associations such as the British Obesity and Metabolic Surgery (BOMSS) in the UK [42]. In our study, patients treated by high volume providers were associated with longer LOS relative to very low providers. This may in part reflect higher volume providers handling a disproportionately larger proportion of more complicated or riskier cases.

Bariatric surgery is considered a generally safe management option for morbidly obese patients, although health outcomes might vary by surgery type, patient characteristics, study period, and setting [43]. In a meta-analysis of cohort studies and clinical trials from Europe, North America, South America, Australia/New Zealand, and Asia, the total mortality at $\leq 30$ days was $0.28 \%(95 \% \mathrm{CI}, 0.22-0.34)$ and total mortality at $\geq 30$ days to 2 years was $0.35 \%(95 \% \mathrm{CI}$, $0.12-0.58$ ) [43]. In the present study, the observed inpatient mortality rate in England $(<0.2 \%)$ was comparable to NBSRreported rates reported across a similar observation period [44]. While banding may be a preferred option given its relatively low LOS, complication, and mortality rate, it may be less effective than GBP and SG with regard to long-term sustained weight loss and may be associated with a higher rate of revision beyond 7-15 years post-implant [14, 34, 45, 46].

\section{Limitations}

The HES database represents an important and useful source of information, but it does have some limitations. Patient-level BMI and weight data were not available in the HES dataset. Thus, we were unable to study sustained weight loss as an end point, nor adjust the LOS, readmission, or complication models for potential differences in BMI between patient groups undergoing each procedure type. Similarly, long-term complication data and post-banding were not available to analyze trends in revisional surgery nor were data available on the long-term impacts of surgery on T2DM. Non-NHSfunded bariatric surgery activities undertaken by independent providers were not available from HES and thus not included in the analysis. While our observational study employed a 
Table 5 Adjusted regression analyses for LOS, 30-day readmission, and post-surgery complication

\begin{tabular}{|c|c|c|c|c|c|c|c|}
\hline & \multicolumn{3}{|c|}{ Length of stay } & \multicolumn{2}{|c|}{ 30-day readmission } & \multicolumn{2}{|c|}{ Post-surgery complication } \\
\hline & $\beta$-estimate & Exponentiated $\beta$ & $95 \% \mathrm{CI}$ & OR & $95 \% \mathrm{CI}$ & OR & $95 \% \mathrm{CI}$ \\
\hline Intercept & 0.5389 & 2.4586 & $(0.5256,0.5522)$ & 0.1694 & $(0.1398,0.2050)$ & 0.0451 & $(0.0322,0.0629)$ \\
\hline \multicolumn{8}{|l|}{ Surgery type ${ }^{a}$} \\
\hline Sleeve gastrectomy (SG) & -0.0094 & 0.0219 & $(-0.0185,-0.0003)$ & 0.9792 & $(0.8579,1.1150)$ & 0.9137 & $(0.7392,1.1211)$ \\
\hline Gastric banding (GB) & -0.0867 & 0.2210 & $(-0.0939,-0.0795)$ & 0.5789 & $(0.5135,0.6517)$ & 0.2746 & $(0.2118,0.3512)$ \\
\hline Other & -0.0098 & 0.0228 & $(-0.0185,-0.0011)$ & 1.2569 & $(1.1188,1.4103)$ & 0.7411 & $(0.5942,0.9171)$ \\
\hline \multicolumn{8}{|l|}{ Individual characteristics } \\
\hline \multicolumn{8}{|l|}{ Age group ${ }^{b}$} \\
\hline $35-44$ years & 0.0002 & 0.0005 & $(-0.0080,0.0084)$ & 0.8680 & $(0.7709,0.9782)$ & 0.9731 & $(0.7927,1.1993)$ \\
\hline $45-54$ years & -0.0004 & 0.0009 & $(-0.0086,0.0078)$ & 0.8999 & $(0.7994,1.0139)$ & 0.9128 & $(0.7413,1.1280)$ \\
\hline $55-64$ years & -0.0071 & 0.0165 & $(-0.0167,0.0025)$ & 0.8128 & $(0.7041,0.9377)$ & 1.0035 & $(0.7876,1.2775)$ \\
\hline $65+$ years & 0.0105 & 0.0245 & $(-0.0102,0.0313)$ & 0.6851 & $(0.4817,0.9486)$ & 0.7490 & $(0.3903,1.3063)$ \\
\hline Female $\operatorname{sex}^{\mathrm{c}}$ & 0.0057 & 0.0132 & $(-0.0008,0.0122)$ & 0.9672 & $(0.8790,1.0655)$ & 1.0634 & $(0.9022,1.2588)$ \\
\hline Non-Caucasian ethnic group ${ }^{\mathrm{d}}$ & -0.0109 & 0.0254 & $(-0.0172,-0.0045)$ & 0.8211 & $(0.7448,0.9044)$ & 0.7143 & $(0.5989,0.8484)$ \\
\hline \multicolumn{8}{|l|}{ Geographical area ${ }^{\mathrm{e}}$} \\
\hline North England & 0.0299 & 0.0713 & $(0.0215,0.0383)$ & 0.8907 & $(0.7893,1.0053)$ & 0.7805 & $(0.6294,0.9683)$ \\
\hline Central England & -0.0743 & 0.1866 & $(-0.0837,-0.0650)$ & 1.1254 & $(0.9833,1.2877)$ & 1.1529 & $(0.9036,1.4685)$ \\
\hline East England & -0.0154 & 0.0361 & $(-0.0294,-0.0015)$ & 0.7668 & $(0.6156,0.9500)$ & 1.1608 & $(0.8528,1.5726)$ \\
\hline South England & -0.0144 & 0.0337 & $(-0.0231,-0.0056)$ & 0.7689 & $(0.6719,0.8796)$ & 1.0543 & $(0.8366,1.3280)$ \\
\hline \multicolumn{8}{|c|}{ Index of multiple deprivation (IMD) ${ }^{\mathrm{f}}$} \\
\hline 2nd & 0.0031 & 0.0072 & $(-0.0045,0.0106)$ & 0.9922 & $(0.8889,1.1071)$ & 0.9726 & $(0.8039,1.1753)$ \\
\hline $3 \mathrm{rd}$ & 0.0044 & 0.0102 & $(-0.0039,0.0128)$ & 0.9038 & $(0.7977,1.0228)$ & 0.8932 & $(0.7192,1.1052)$ \\
\hline 4th & 0.0158 & 0.0371 & $(0.0068,0.0248)$ & 0.8781 & $(0.7649,1.0062)$ & 0.8290 & $(0.6503,1.0497)$ \\
\hline 5th quintile (least deprived) & 0.0025 & 0.0058 & $(-0.0077,0.0127)$ & 0.9257 & $(0.7918,1.0790)$ & 0.9005 & $(0.6884,1.1672)$ \\
\hline \multicolumn{8}{|l|}{ Clinical characteristics } \\
\hline Independent provider ${ }^{\mathrm{g}}$ & -0.0616 & 0.1524 & $(-0.0761,-0.0471)$ & 0.9803 & $(0.7940,1.2031)$ & 0.6966 & $(0.4570,1.0278)$ \\
\hline \multicolumn{8}{|l|}{ Procedure volume $\mathrm{h}^{\mathrm{h}}$} \\
\hline Low & -0.0080 & 0.0186 & $(-0.0166,0.0006)$ & 0.9384 & $(0.8282,1.0635)$ & 1.0709 & $(0.8620,1.3324)$ \\
\hline Medium & -0.0404 & 0.0975 & $(-0.0486,-0.0322)$ & 0.8992 & $(0.7962,1.0158)$ & 0.6176 & $(0.4883,0.7802)$ \\
\hline High & 0.0720 & 0.1803 & $(0.0627,0.0813)$ & 0.7843 & $(0.6838,0.8991)$ & 1.0414 & $(0.8313,1.3051)$ \\
\hline \multicolumn{8}{|c|}{ Charlson comorbidity index $(\mathrm{CCI})^{\mathrm{i}}$} \\
\hline Moderate (1-5) & -0.0046 & 0.0106 & $(-0.0108,0.0016)$ & 1.0802 & $(0.9836,1.1855)$ & 1.4773 & $(1.2648,1.7239)$ \\
\hline High $(\geq 6)$ & 0.0015 & 0.0035 & $(-0.0091,0.0122)$ & 1.6485 & $(1.4330,1.8908)$ & 1.8661 & $(1.4792,2.3333)$ \\
\hline Adjusted $R$ square $^{\mathrm{j}}$ & 0.2777 & & & 0.0067 & & 0.0143 & \\
\hline
\end{tabular}

Reference group

${ }^{a}$ Surgery type, gastric bypass (GBP)

${ }^{\mathrm{b}}$ Age group, 18-34 years

${ }^{\mathrm{c}}$ Sex, men

${ }^{\mathrm{d}}$ Ethnic group, Caucasian

${ }^{\mathrm{e}}$ Geographical area, London

${ }^{\mathrm{f}}$ Index of multiple deprivation, 1st quintile (most deprived)

${ }^{\mathrm{g}}$ Provider type, NHS trust

${ }^{\text {h }}$ Procedure volume, very low (1-399)

${ }^{\mathrm{i}}$ Charlson comorbidity score, no comorbidity $(0)$

${ }^{\mathrm{j}}$ Adjusted $R^{2}$ for model with LOS as outcome, adjusted McFadden pseudo $R^{2}$ for model with 30-day readmission and post-surgery complication as outcomes 
retrospective design, the distribution of key patient demographic and clinical characteristics including comorbidities and provider factors were generally well balanced across each of the procedure types considered. There was thus no obvious signal suggesting a marked selection bias secondary to healthier patients being preferentially selected for any one particular type of procedure. Finally, the small number of mortality events prohibited any adjusted analysis secondary to underpowering, while utilization trends were not analyzed post 2011.

In conclusion, gastric bypass was the most frequently reported procedure in England across the observation period. While utilization across all procedure types increased between 2007 and 2010, overall uptake of bariatric surgery in England represents only a small proportion of the eligible population. Readmission and complication rates were lower for gastric banding relative to those for either gastric bypass or sleeve gastrectomy. The observed inpatient mortality rate was low across all procedure types.

Source of Funding The study was supported by Covidien Inc. (now part of Medtronic). The sponsor has no ultimate authority over any of the following activities: study design, data collection, management, analysis and interpretation of data, writing the report, and the decision to submit the report for publication.

\section{Compliance with Ethical Standards}

Conflict of Interest All authors have completed the ICMJE uniform disclosure form and declare that three of the authors are employees of Synergus AB - MedTech consulting company, which received a grant from Covidien Inc. (now part of Medtronic) to perform the study.

Ethical Consideration All analyses were presented at the group level, and no individual can be traced. Data were obtained from HES and complied with HES requirements. No ethical permissions were required for such analysis in the UK

Open Access This article is distributed under the terms of the Creative Commons Attribution 4.0 International License (http:// creativecommons.org/licenses/by/4.0/), which permits unrestricted use, distribution, and reproduction in any medium, provided you give appropriate credit to the original author(s) and the source, provide a link to the Creative Commons license, and indicate if changes were made.

\section{References}

1. World Health Organization regional office for Europe. Obesity: data and statistics [Internet]. 2015. Available from: http://www. euro.who.int/en/health-topics/noncommunicable-diseases/obesity/ data-and-statistics

2. World Health Organization (WHO). Global Health Observatory data repository (GHO) [Internet]. GHO. [cited 2016 Aug 30]. Available from: http://gamapserver.who.int/gho/interactive charts/ ncd/risk factors/obesity/atlas.html

3. National Health Services UK. Health survey for England, 2014: trend tables [NS] [Internet]. 2016 [cited 2016 Aug 26]. Available from: http://digital.nhs.uk/catalogue/PUB19297
4. OECD. Obesity update 2014 [Internet]. Available from: http:// www.oecd.org/els/health-systems/Obesity-Update-2014.pdf

5. Yumuk V, Tsigos C, Fried M, et al. European guidelines for obesity management in adults. Obesity Facts. 2015;8:402-24.

6. Abdullah A, Peeters A, de Courten M, et al. The magnitude of association between overweight and obesity and the risk of diabetes: a meta-analysis of prospective cohort studies. Diabetes Res Clin Pract. 2010;89:309-19.

7. Huerta JM, Tormo M-J, Chirlaque M-D, et al. Risk of type 2 diabetes according to traditional and emerging anthropometric indices in Spain, a Mediterranean country with high prevalence of obesity: results from a large-scale prospective cohort study. BMC Endocr Disord. 2013;13:7.

8. Strazzullo P, D'Elia L, Cairella G, et al. Excess body weight and incidence of stroke: meta-analysis of prospective studies with 2 million participants. Stroke. 2010;41:e418-26.

9. Nguyen NT, Magno CP, Lane KT, et al. Association of hypertension, diabetes, dyslipidemia, and metabolic syndrome with obesity: findings from the National Health and Nutrition Examination Survey, 1999 to 2004. J Am Coll Surg. 2008;207:928-34.

10. Renehan AG, Soerjomataram I, Tyson M, et al. Incident cancer burden attributable to excess body mass index in 30 European countries. Int J Cancer. 2010;126:692-702.

11. National Institute for Health and Care Excellence (NICE). Obesity: guidance on the prevention of overweight and obesity in adults and children [Internet]. 2014 [cited 2015 Jan 1]. Available from: https:// www.nice.org.uk/guidance/cg43

12. Diamantis T, Apostolou KG, Alexandrou A, et al. Review of longterm weight loss results after laparoscopic sleeve gastrectomy. Surg Obes Relat Dis. 2014;10:177-83.

13. Picot J, Jones J, Colquitt JL, Gospodarevskaya E, Loveman E, Baxter L, et al. The clinical effectiveness and cost-effectiveness of bariatric (weight loss) surgery for obesity: a systematic review and economic evaluation. Health Technol Assess. 2009;13:1-190, 215 357, iii-iv.

14. Chang SH, Stoll CR, Song J, et al. The effectiveness and risks of bariatric surgery: an updated systematic review and meta-analysis, 2003-2012. JAMA Surg. 2014;149:275-87.

15. Colquitt JL, Pickett K, Loveman E, Frampton GK. Surgery for weight loss in adults. Cochrane Database of Systematic Reviews [Internet]. John Wiley \& Sons, Ltd; 2014 [cited 2017 Feb 6]. Available from: http://onlinelibrary.wiley.com.proxy.kib.ki.se//10. 1002/14651858.CD003641.pub4/abstract

16. Keating CL, Dixon JB, Moodie ML, et al. Cost-efficacy of surgically induced weight loss for the management of type 2 diabetes: a randomized controlled trial. Diabetes Care. 2009;32:580-4.

17. Ribaric G, Buchwald J, McGlennon T. Diabetes and weight in comparative studies of bariatric surgery vs conventional medical therapy: a systematic review and meta-analysis. Obes Surg. 2014;24:437-55.

18. Ells LJ, Macknight MN, Wilkinson JR. Obesity surgery in England: an examination of the health episode statistics 1996-2005. Obes Surg. 2007;17:400-5.

19. The National Bariatric Surgery Registry (NBSR). 2014 National Bariatric Surgery Registry (NBSR Report) [Internet]. [cited 2016 Aug 26]. Available from: http://nbsr.co.uk/2014-report/

20. Health and Social Care Information Centre (HSCIC). Statistics on obesity, physical activity and diet: England 2015 [Internet]. 2015 Mar. Available from: http://content.digital.nhs.uk/catalogue/ PUB16988/obes-phys-acti-diet-eng-2015.pdf

21. Yearly Report Scandinavian Obesity Surgery Registry (SOReg) 2015: Part I operation statistics and early complication. 2016 May.

22. Morino M, Toppino M, Forestieri P, et al. Mortality after bariatric surgery: analysis of 13,871 morbidly obese patients from a national registry. Ann Surg. 2007;246:1002-9. 
23. Smith MD, Patterson E, Wahed AS, et al. 30-Day mortality after bariatric surgery: independently adjudicated causes of death in the longitudinal assessment of bariatric surgery. Obes Surg. 2011;21: 1687-92.

24. Centre H\& SC information. Hospital Episode Statistics [Internet]. 2015. Available from: http://www.hscic.gov.uk/hes

25. ICD-10: international statistical classification of diseases and related health problems. Geneva: World Health Organization; 2011.

26. Office of the Deputy Prime Minister DC. The English Indices of Deprivation 2004: summary (revised) [Internet]. 2011. Available from: https://www.dorsetforyou.com/media/162073/ Index-of-Deprivation-2010-report/pdf/Index_of_Deprivation 2010 report.pdf

27. Burns EM, Naseem H, Bottle A, et al. Introduction of laparoscopic bariatric surgery in England: observational population cohort study. BMJ. 2010;341:c4296.

28. Birkmeyer JD, Sun Y, Goldfaden A, et al. Volume and process of care in high-risk cancer surgery. Cancer. 2006;106:2476-81.

29. Goode SD, Keltie K, Burn J, et al. Effect of procedure volume on outcomes after iliac artery angioplasty and stenting: effect of procedure volume on outcomes after iliac artery interventions. Br J Surg. 2013;100:1189-96.

30. Clinical Indicators Team. Indicator specification: summary hospital-level mortality indicator. Health \& Social Care Information Centre; 2015 Aug. Report No.: I00699 1.20.

31. R Core Team. R: a language and environment for statistical computing. [Internet]. Vienna, Austria: R Foundation for Statistical Computing; 2015. Available from: https://www.R-project.org/.

32. Ahmad A, Laverty AA, Aasheim E, et al. Eligibility for bariatric surgery among adults in England: analysis of a national crosssectional survey. Journal of the Royal Society of Medicine Open. 2014;5:1-6.

33. Borisenko O, Colpan Z, Dillemans B, et al. Clinical indications, utilization, and funding of bariatric surgery in Europe. Obes Surg. 2015;25:1408-16.

34. O'Brien PE, MacDonald L, Anderson M, et al. Long-term outcomes after bariatric surgery: fifteen-year follow-up of adjustable gastric banding and a systematic review of the bariatric surgical literature. Ann Surg. 2013;257:87-94.
35. Runkel N, Colombo-Benkmann M, Hüttl TP, et al. Evidencebased German guidelines for surgery for obesity. Int J Color Dis. 2011;26:397-404.

36. Robert M, Pasquer A, Espalieu P, et al. Gastric bypass for obesity in the elderly: is it as appropriate as for young and middle-aged populations? Obes Surg. 2014;24:1662-9.

37. Kathryn L, Kemmeter PR, Kemmeter KD. Bariatric surgery outcomes in patients aged 65 years and older at an American Society for Metabolic and Bariatric Surgery Center of Excellence. Obesity Surgery. 2010;20:1199-205.

38. Melissas J. IFSO guidelines for safety, quality, and excellence in bariatric surgery. Obes Surg. 2008;18:497-500.

39. DeMaria EJ, Pate V, Warthen M, et al. Baseline data from American Society for Metabolic and Bariatric Surgery-designated Bariatric Surgery Centers of Excellence using the Bariatric Outcomes Longitudinal Database. Surg Obes Relat Dis. 2010;6:347-55.

40. Champion JK, Pories WJ. Centers of Excellence for bariatric surgery. Surg Obes Relat Dis. 2005;1:148-51.

41. Pratt GM, McLees B, Pories WJ. The ASBS Bariatric Surgery Centers of Excellence program: a blueprint for quality improvement. Surg Obes Relat Dis. 2006;2:497-503.

42. BOMSS standards for clinical services | BOMSS [Internet]. [cited 2016 Oct 18]. Available from: http://www.bomss.org.uk/bomssstandards-for-clinical-services/

43. Buchwald H, Estok R, Fahrbach K, et al. Trends in mortality in bariatric surgery: a systematic review and meta-analysis. Surgery. 2007;142:621-35.

44. The National Bariatric Surgery Registry (NBSR). 2010 National Bariatric Surgery Registry (NBSR) report [Internet]. [cited 2016 Aug 26]. Available from: http://nbsr.co.uk/2010-report/

45. Schiavo L, Scalera G, Barbarisi A. Sleeve gastrectomy to treat concomitant polycystyc ovary syndrome, insulin and leptin resistance in a 27 -years morbidly obese woman unresponsive to insulin-sensitizing drugs: a 3-year follow-up. Int J Surg Case Rep. 2015;17:36-8.

46. By-Band-Sleeve Study. By-Band-Sleeve study [Internet]. [cited 2016 Nov 23]. Available from: http://www.by-band-sleeve. bristol.ac.uk/ 